\title{
Ancient T-independence of mucosal IgX/A: gut microbiota unaffected by larval thymectomy in Xenopus laevis
}

\author{
S Mashoof ${ }^{1}$, A Goodroe ${ }^{1}$, CC Du ${ }^{1}$, JO Eubanks ${ }^{1}$, N Jacobs ${ }^{1}$, JM Steiner ${ }^{2}$, I Tizard $^{3}$, JS Suchodolski ${ }^{2}$ and \\ MF Criscitiello ${ }^{1}$
}

Many studies address the influence of the gut microbiome on the immune system, but few dissect the effect of T cells on gut microbiota and mucosal responses. We have employed larval thymectomy in Xenopus to study the gut microbiota with and without the influence of T lymphocytes. Pyrosequencing of $16 \mathrm{~S}$ ribosomal RNA genes was used to assess the relative abundance of bacterial groups present in the stomach, small and large intestine. Clostridiaceae was the most abundant family throughout the gut, while Bacteroidaceae, Enterobacteriaceae, and Flavobacteriaceae also were well represented. Unifrac analysis revealed no differences in microbiota distribution between thymectomized and unoperated frogs. This is consistent with immunization data showing that levels of the mucosal immunoglobulin IgX are not altered significantly by thymectomy. This study in Xenopus represents the oldest organisms that exhibit class switch to a mucosal isotype and is relevant to mammalian immunology, as IgA appears to have evolved from IgX based upon phylogeny, genomic synteny, and function.

\section{INTRODUCTION}

The thymus is the primary T-lymphoid organ of vertebrates from sharks to mammals. ${ }^{1,2}$ In humans, a small deletion on chromosome 22 in DiGeorge syndrome often results in an absent or hypoplastic thymus, with resulting loss of T-mediated responses (reviewed in ref. 3). The hairless "nude" strain of mouse has an absent or greatly degenerated thymus owing to a mutation in the Foxn 1 gene. ${ }^{4}$ These mice do have B cells but $\mathrm{T}$ cells are very few. Owing to the lack of both cytotoxic and helper $\mathrm{T}$ cells, nude or thymectomized mice have abolished allograft and mixed leukocyte reactions, proliferative responses to classical T-cell mitogens, and antibody responses against T-dependent antigens. ${ }^{5}$

The mucosal immune system forms the largest vertebrate immune compartment and is mediated by specialized cells and immunoglobulins, such as plasma cells producing secretory IgA in birds and mammals. IgA production in the gut is not constitutive, shown by the absence of both $\operatorname{IgA}$ and IgA-secreting plasma cells in the lamina propria of germ-free mice. ${ }^{6}$ There are many studies investigating the humoral mucosal immune responses of mammals lacking $\mathrm{T}$ cells, but they have yielded mixed results. ${ }^{7,8}$ However, a picture is emerging of a significant T-cell-independent mechanism of gut IgA management of mutualistic flora. ${ }^{9,10}$ Gut IgA-producing plasma cells in mammals employ tumor necrosis factor- $\alpha$ and inducible nitric oxide synthase usually associated with innate phagocytes. ${ }^{11}$ Interestingly, B cells of lower vertebrates have been found to have strong phagocytic activity. ${ }^{12}$ The B-cell phagocytic activity is consistent with an emerging theme of primitive, innate, T-independent, IgA-switched B cells in the gut, although this has never been actually tested in a lower tetrapod. This gap in our knowledge prompted our assessment of the T-dependence of humoral mucosal immunology in a phylogenetically relevant model species.

The African clawed frog Xenopus laevis belongs to the tongue-less frog family Pipidae. It is a choice model for ontogeny and phylogeny of both humoral and cell-mediated immunity. Xenopus shares a common ancestor with 
a

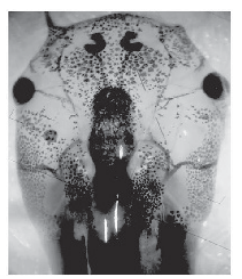

b

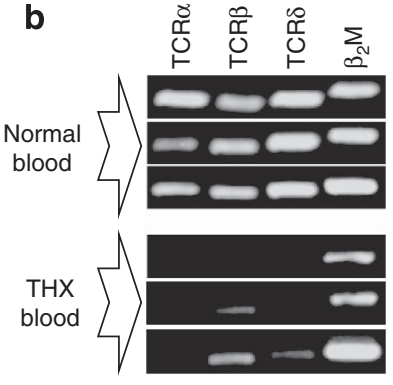

C

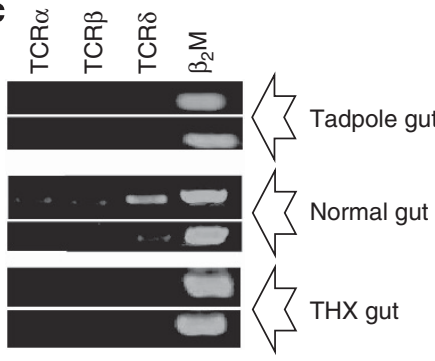

Figure 1 Larval thymectomy greatly depletes TCR $\alpha$ and $\delta$ message from the adult frogs. (a) Unilateral thymectomy at day 9 shows absence of naturally melanized thymus on the left compared with intact organ on the right. Experimental frogs were bilaterally thymectomized. Image captured at day 20 at original magnification $\times 3$. (b) PCR contrasting levels of TCR $\alpha, \beta$, and $\delta$ to $\beta_{2}$-microglobulin in peripheral blood of three intact postmetamorphic frogs and three thymectomized post-metamorphic frogs. Results of 40 cycles of amplification. (c) Reverse transcriptase PCR comparing the same amplicons from intestine of 9-day tadpoles (the age of thymectomy, gut of 10 animals pooled per row) and two intact post-metamorphic frogs and two thymectomized post-metamorphic frogs.

mammals 350 million years ago and links them to the more ancient vertebrates where the adaptive immune system arose (reviewed in ref. 13). The ability to perform thymectomy on transparent early stage Xenopus tadpoles made this frog an ideal model species to query the thymic-dependent management of gut microbiota and mucosal antibody responses from a fundamental point in vertebrate humoral immunity (reviewed in ref. 14).

This present study is the first investigation determining the gut bacterial populations of an amphibian using 454 pyrosequencing of the $16 \mathrm{~S}$ ribosomal RNA (rRNA) gene. In addition, we examined the flora of normal and thymectomized frogs, and the ability of thymectomized frogs to make mucosal antibody responses. $\operatorname{IgX}$ has been functionally associated with mucosal responses and (in contrast to IgY) was found in thymectomized frogs. ${ }^{15,16}$ However, evolutionarily it has been thought to be closer to IgM. ${ }^{17}$ T-independent responses are known from Xenopus serum, ${ }^{18}$ but here we aimed to determine the effect of thymectomy upon the gut flora, the mucosal and the systemic IgX responses. We also evaluated the relationship of amphibian IgX to mammalian IgA, in hopes of resolving ambiguity as to the origins and natural history of the class of antibody that manages vast numbers of mutualistic microbes and is the first defense of the barriers breached by most pathogens.

\section{RESULTS}

\section{Larval thymectomy depletes $\mathrm{T}$ cells in the adult}

To study the role of $\mathrm{T}$ cells in the management of gut bacterial communities and mucosal humoral immunity from a fundamental standpoint in vertebrate evolution, we used the frog larval thymectomy model. We performed thymectomies largely executed as in the original studies, ${ }^{19}$ but 2 days later in development than in the original protocol owing to slightly slower development of the larvae in our system. No frogs used in the thymectomized group showed any thymic regrowth after cauterization (using microscopic inspection). Thymectomy greatly diminished the detectable number of TCR $\alpha$ transcripts using constant region PCR in the adult frog. TCR $\beta$ and $\delta$ amplicons could be detected in some frogs, but at a much lower frequency than in frogs that had not undergone surgery (Figure 1b). Regardless, larval bilateral thymectomy almost completely ablated expression of TCR $\alpha$, showing that the classic $\alpha \beta$ T-cell compartment has been removed from the experimental gut model.

\section{Pyrosequencing reveals diverse gut bacterial flora in amphibian}

DNA was prepared from frog luminal contents at three distinct locations in the gastrointestinal tract, which is relatively simple in poikilothermic frogs and reptiles as they have lower metabolic rates compared with most mammals and birds (Supplementary Figure S1 online). Pyrosequencing of the 16S rRNA gene resulted in a total of 51,992 quality-sequencing tags (mean, range: 2,888, 1,870-5,314). Figure 2 illustrates the rarefaction curves for all samples at 1,800 sequences, suggesting sufficient coverage. Although rare operational taxonomic units may have been identified with a higher sequencing depth, the number of sequence tags used here is deemed adequate to allow comparison of the beta diversity between samples. ${ }^{20}$ Samples taken from large intestine showed the richest diversity of flora. Individual frogs showed distinct microbiota composition (Figure 3). Four frogs yielded similar rarefaction curves, whereas one non-thymectomized frog (N321) showed more diversity and one non-thymectomized (N571) less. Averaged thymectomized samples gave a very similar rarefaction plot as those from normal animals. These data allowed us to gain an initial molecular description of the base amphibian gastrointestinal microbiota and look for differences between anatomic sites and surgical groups.

\section{Frog gut flora is anatomically distinct but not altered by T cells}

Many of the prokaryotic groups that dominate the human flora are also major components in the frog flora. Clostridiaceae dominated this amphibian community, and Bacteroidaceae and Enterobacteriaceae were abundant in our sequencing (Figure 3, Supplementary Table 3 online). In contrast to terrestrial mammalian flora, the environmental Flavobacteriaceae 

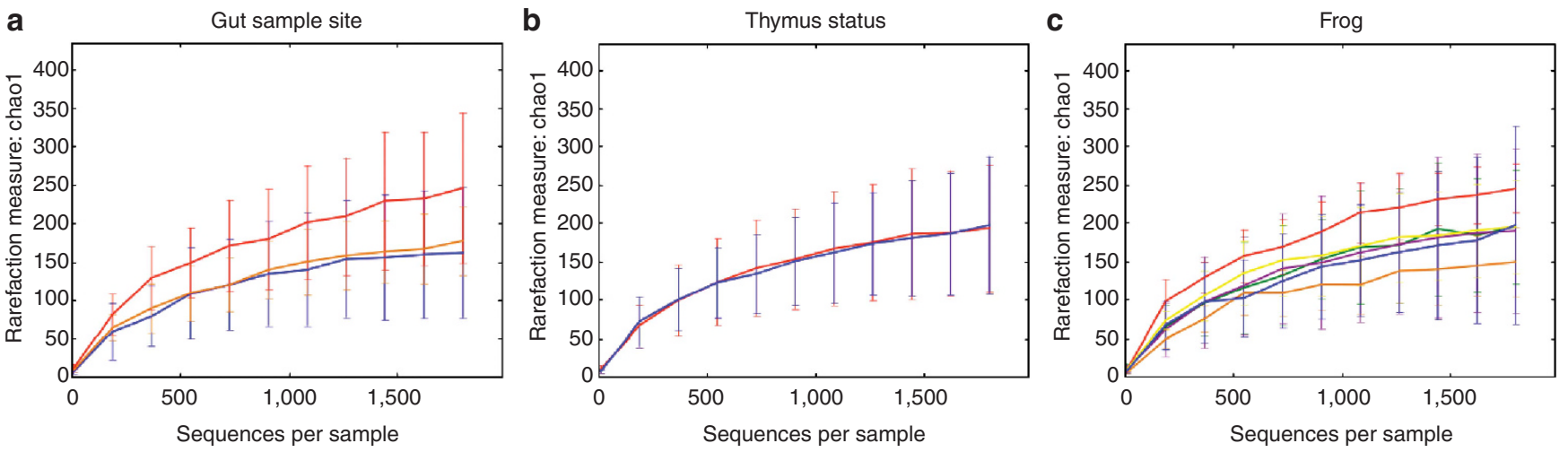

Figure 2 Rarefaction analysis of 16S ribosomal RNA gene sequences obtained from frog gastrointestinal content. The analysis was performed on a randomly selected subset of 1,800 sequences per sample. Lines represent the average of each sample type. (a) Stomach, orange; large intestine, red; and small intestine, blue. (b) Normal (no surgery), blue and thymectomized, red. (c) Frog N011, red; N321, blue; T258, green; T606, yellow; T339, brown; and N571, orange.

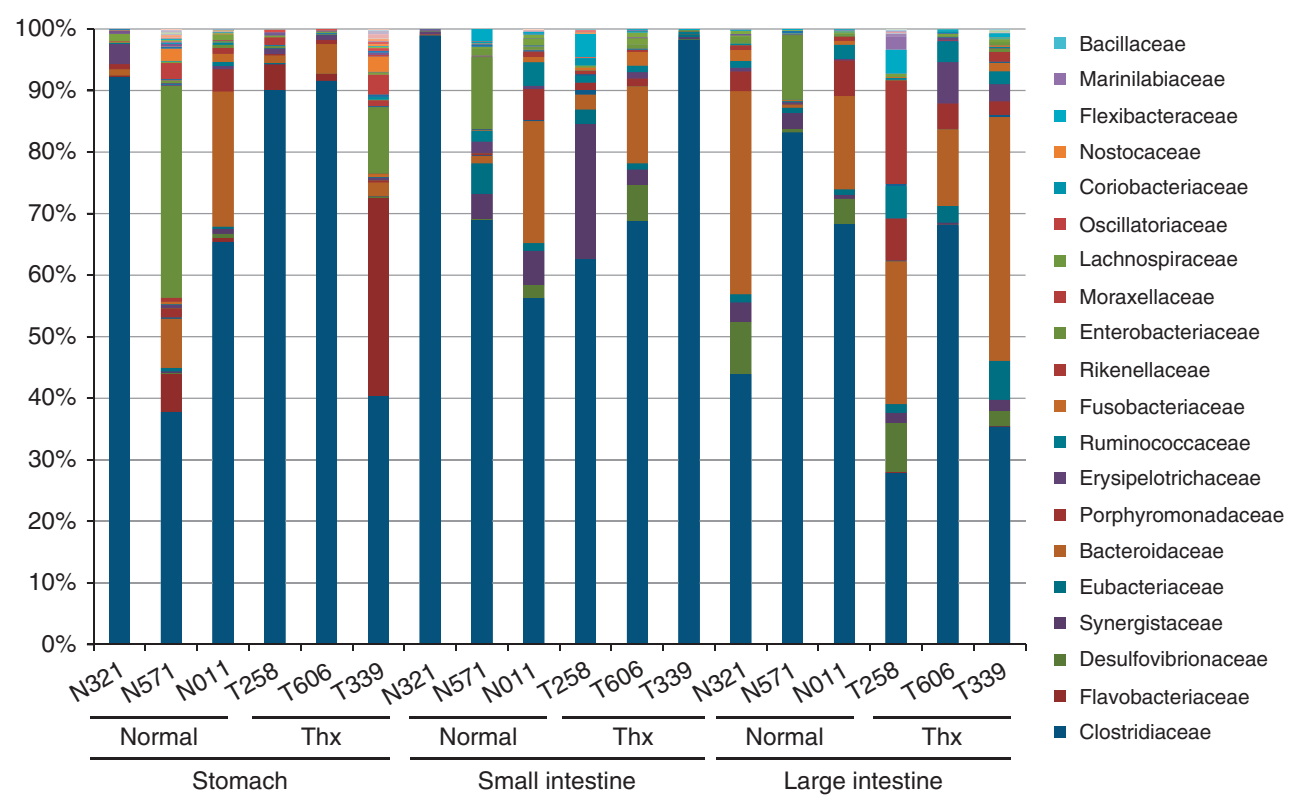

Figure 3 Bacterial families in the gastrointestinal tract of $X$. laevis. Familial distribution is shown with different colors from individual samples, grouped by thymus status from each of the three sampled positions in the gastrointestinal tract. Families with at least $1 \%$ representation in any sample are listed at the right. Complete taxonomic data is in Supplementary Table $\mathbf{S 2}$ online. Clostridiaceae were the predominant family, and no differences in bacterial groups between normal and thymectomized frogs were observed.

constituted nearly a third (32.34\%) of the stomach flora of one frog and comprised 6.20 and $4.20 \%$ in two other individuals. The Synergistaceae, Desulfovibrionaceae, Erysipelotrichaceae, Ruminococcaceae, Rikenellaceae, and Porphyromonadaceae also were substantial contributors (over $5 \%$ in at least one sample) to the $X$. laevis microbiota.

The principal coordinates analysis plots based on the unweighted UniFrac distance metric indicated that the $X$. laevis stomach is composed of distinct microbial communities compared with the small and large intestine (Figure 4a). This was most pronounced in the greater representation of Flavobacteriaceae in the stomach compared with the more distal sites. Oscillatoriaceae cyanobacteria and Enterobacteriaceae were in greater abundance in the stomach. This latter group includes the common Gram-negative and sometimes pathogenic Salmonella, Escherichia coli, Klebsiella, Shigella, and Yersinia pestis more commonly associated with the mammalian intestine. Synergistaceae was found in the intestines more than the stomach, particularly in the small intestine of thymectomized frog \#258. However, principal coordinates analysis plots based on the unweighted UniFrac did not reveal differences between small and large intestinal microbiota and, importantly, between normal and thymectomized frogs (Figure $4 b$ ). The similar microbiological findings in the guts of normal and 

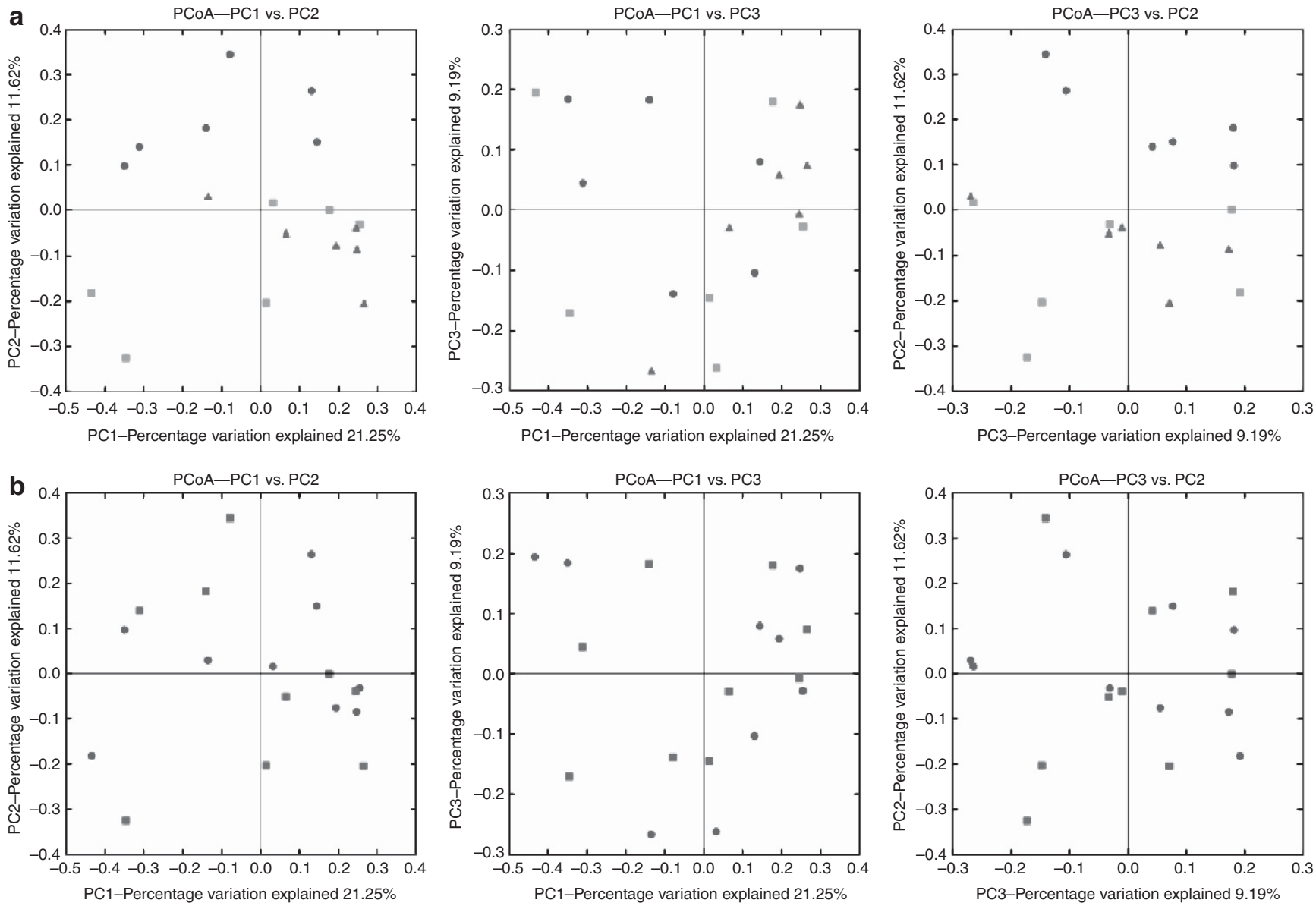

Figure 4 Principal coordinates analysis (PCoA) of unweighted UniFrac distances of 165 ribosomal RNA gene sequencing. The analysis was performed on a randomly selected subset of 1,800 sequences per sample. (a) Stomach (blue circles) samples separated from small (green square) and large (red triangle) intestine, indicative of distinct flora. (b) No such separation was seen between non-thymectomized (blue circle) and thymectomized (red square) samples.

T-cell-depleted frogs prompted investigations of the mucosal humoral immune compartment in this model.

\section{Amphibian IgX is likely orthologous to mammalian IgA}

To assess the T-dependence of humoral immunity in the frog alimentary canal, we wanted to be more confident of the mucosal immunoglobulin in this amphibian. As more immunogenetic data have recently become available from reptile, bird, and ancestral groups of mammals (Supplementary Table S3 online), we revisited the phylogenetic relationships of tetrapod antibody classes to see if there was now convergence of expression and functional data suggesting $\operatorname{IgX}$ as the mucosal isotype. Entire immunoglobulin heavy chain $\mathrm{C}$ region sequences from diverse vertebrates were used to determine the relationship between amphibian IgX and mammalian IgA (Figure 5). Sequences were aligned and manually adjusted to maintain domain alignment between isotypes having either three or four constant domains (Supplementary Figure S3 online). The resulting trees showed that IgX did not cluster closest to IgM. Unlike any past phylogenetic analyses, these data show that $\operatorname{IgX}$ and $\operatorname{IgA}$ share a common ancestor earlier than either IgX or IgA does with IgM with high statistical support ( $91 \%$ of 1,000 bootstrap replications). This finding provided confidence in assaying IgX in the frog as an ortholog as well as a functional analog of mammalian IgA in mucosal immunity.

\section{Thymectomy does not impede mucosal antibody production}

Normal and thymectomized frogs distinct from those assayed for gut flora were immunized with dinitrophenol-keyhole limpet hemocyanin (DNP-KLH) either intracoelomically (IC) or orally (Figure 6). B cells were harvested and cultured from the spleen and gut of these animals, and enzyme-linked immunosorbent assays (ELISAs) were performed for both total IgX and antigen-specific IgX on the supernatant. Oral immunization elicited significantly $(P=0.025)$ more total IgX from intestinal B cells than IC delivery, but no significant difference was seen from the spleen cells or between B cells from normal and thymectomized animals. When DNP-KLH-specific IgX was assayed, the only significant $(P=0.013)$ difference seen was an increase in specific IgX from orally immunized splenocytes from thymectomized frogs vs. orally immunized spleen cells from normal frogs. Thus larval thymectomy does not appear to retard the frogs' ability to make total IgX or IgX specific to this hapten-carrier conjugate. 


\section{ARTICLES}

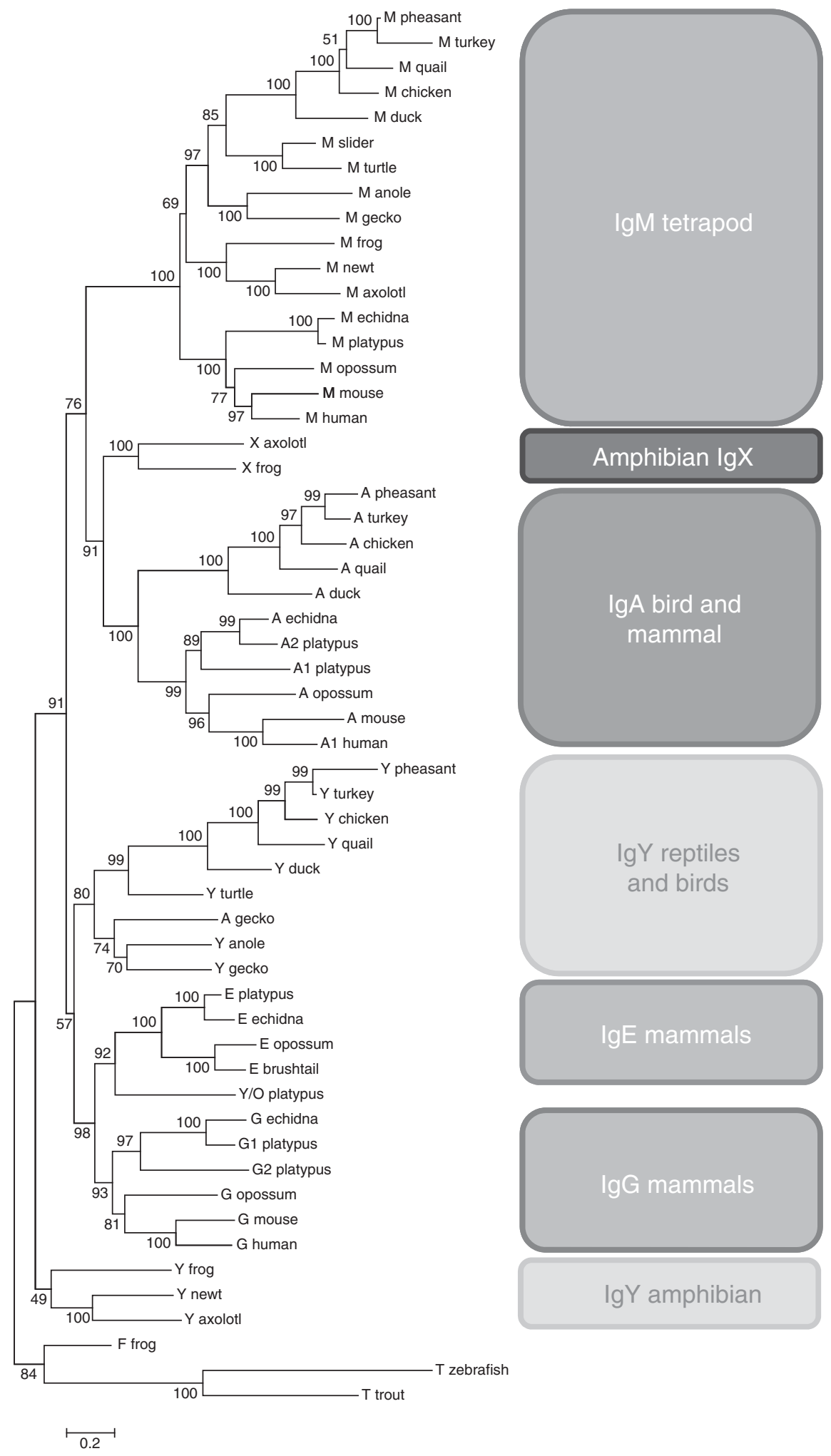

Figure 5 Amphibian $\lg X$ is orthologous to $\lg A$ of birds and mammals. Neighbor-joining phylogenetic tree of the constant regions of diverse tetrapod immunoglobulin heavy chains, with the fish mucosal isotype lgZ/T, included as an outgroup. Numbers at nodes show bootstrap support for each bifurcation after 1,000 replications. The alignment used to build this is available as Supplementary Figure S2 online. 
a

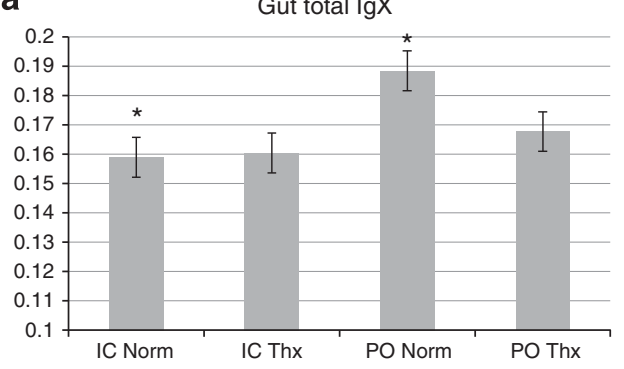

C

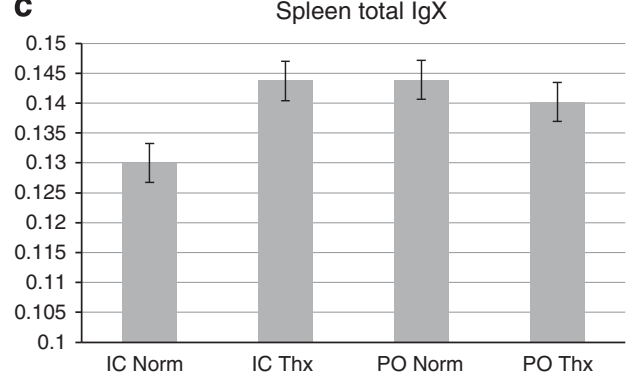

b

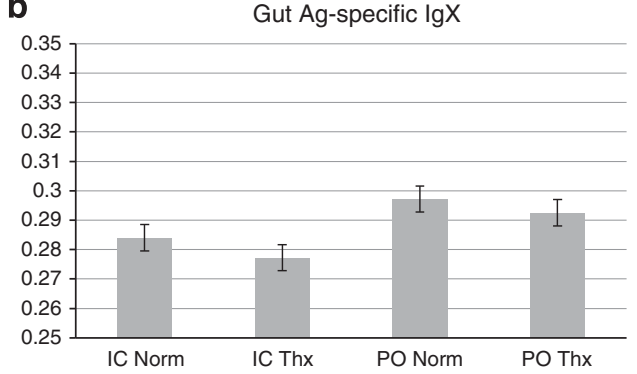

d

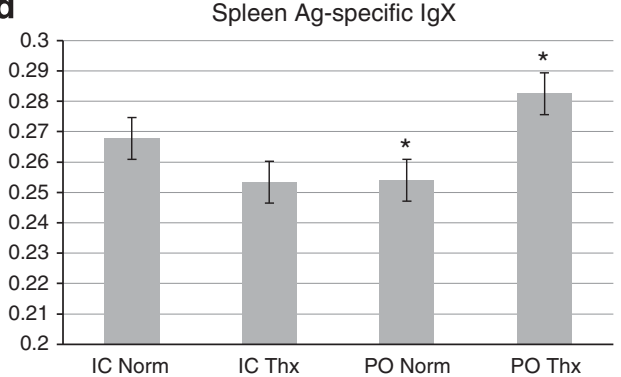

Figure 6 Thymectomy does not retard induction of mucosal IgX response. Enzyme-linked immunosorbent assay for the $\lg X$ isotype on supernatant of lymphocytes cultured from the spleen or intestine of frogs immunized to dinitrophenol-keyhole limpet hemocyanin. Units of the Y-axes are absorbance at $450 \mathrm{~nm}$. (a) Oral (p.o.) gives a significantly greater total $\lg X$ response in the gut than intracoelomic (IC) immunization ( $P=0.025$, marked by *), but no significant difference was seen with thymectomized frogs. No significance was seen monitoring neither antigen-specific lgX in the gut (b) nor total $\lg X$ in the spleen (c). (d) Antigen-specific $\lg X$ actually increased from spleen cells with thymectomy $\left(P=0.013\right.$, marked by ${ }^{*}$.

\section{DISCUSSION}

\section{Frog gut flora}

Molecular sequencing techniques have surpassed culture methods of investigating gastrointestinal microbiota owing to their increased sensitivity (and the majority of unculturable genera present there). ${ }^{21}$ The amplification and subsequent sequencing of the $16 \mathrm{~S}$ rRNA gene allows the identification of bacterial groups present in the gastrointestinal tract of humans and other animal species. ${ }^{22-24}$

High-throughput sequencing techniques had not been applied to the gut microbiota in any amphibian model, and X. laevis's use in developmental, cell- and immunobiology made it an obvious first candidate. ${ }^{25}$ High-throughput $16 \mathrm{~S}$ rRNA sequencing has been used to analyze the antifungal cutaneous bacterial populations in a salamander. ${ }^{26}$ The most comprehensive culture-based studies of amphibian gut flora have been performed in the leop$\operatorname{ard}$ frog (Rana pipiens). ${ }^{27}$ Similar to the present work in Xenopus, Rana was found to have many Clostridiaceae, Eubacteriaceae, and Bacteroidaceae, and hibernating frogs at lower temperatures had a significant shift of flora to dominant Pseudomonodaceae. ${ }^{28}$ Using the pyrosequencing-based approach described here, we were able to identify $>60$ families of bacteria in the Xenopus gut that to our knowledge have not been described previously in amphibians. These include the known decomposers of plant polymers Marinilabiaceae, ${ }^{29}$ the potential pathogens in the guts of humans and fish Porphyromonadaceae, ${ }^{30}$ insect endosymbiont Sphingobacteriaceae, ${ }^{31}$ and the known fermenters of fish microbiota Verrucomicrobiaceae. ${ }^{32}$

The frogs in our gut flora analysis all spent their lives in the same aquatic animal room descended from the same outbred founders just one generation before. They all received the same prepared diet. Although not sterilized, this homogenous, consistent feed is certainly in stark contrast to the varied, inconsistent, and microbe-rich diet wild Xenopus would consume in Africa. Yet, we saw great individual variance in their gastrointestinal flora, as has been described in humans ${ }^{33}$ and dogs. ${ }^{34}$ These communities are undoubtedly temporally dynamic as well within the individual animals. This work in X. laevis provides a reference for anatomically discrete gut microbial communities in an omnivorous amphibian. This is the class of vertebrates that gave rise to the amniotic reptiles, birds, and mammals, and first employed immunoglobulin heavy chain isotype switching to a mucosal isotype.

\section{T-cell influence on mucosal immunity}

Formative studies in nude (athymic) mice found that the poorly developed Peyer's patches lacking substantial germinal centers and low IgA levels of this rodent model could be largely restored by thymic grafts and the resulting T-cell population. ${ }^{35}$ Yet, loss of T-cell function was not found to dramatically alter the cultivable gastrointestinal microbiota in these mice. ${ }^{36}$ As our understanding of T-cell help, class switch recombination and mucosal immunity have improved in the decades since this work; much energy has focused on the relationship between the adaptive immune system and the gut flora. This has extended to hypotheses of the two coevolving and even rationale for the original genesis of the adaptive system. ${ }^{37,38}$

We turned to the major biological model "between" zebrafish and mammals to ask questions about the influence of $\mathrm{T}$ cells in the mucosal immune compartment. This choice affords a 
comparative view of what the first immune system with both major histocompatibility complex-restricted $\mathrm{T}$ cells and a humoral response capable of class switch to a mucosal isotype was like in the ancestral tetrapod 300 million years ago. Thymectomy in this animal is an established model system for removal of the T-cell compartment, ${ }^{14,39-42}$ but this study is the first to rigorously test the adults for T-cell receptor expression by PCR. We assayed TCR $\beta, \delta$, and $\alpha$, as TCR $\gamma$ has been shown to be expressed early in the X. laevis thymus. ${ }^{43}$ Initially, we were dismayed by some constant region (not necessarily indicative of functional rearrangement) TCR messenger RNA expression from peripheral blood of adults in which we visually scored the thymectomies to be perfectly clean. But perhaps this is to be expected, as TCR $\beta$ constant region message has been shown from bone marrow-derived lymphocytes ${ }^{44}$ and even functional TCR $\delta$ and TCR $\gamma$ transcripts have been found in nude mice. ${ }^{45}$ Despite these observed low levels of constant domain nucleic acid expression, we are confident that the scrupulous culling of tadpoles with incomplete surgeries and molecular diagnostics of mature animals yielded frogs with no functional $\alpha \beta$ T-cell compartment (and $\gamma \delta$ as well). Monoclonal antibodies have confirmed this absence at the cellular level of receptor expression in this model. ${ }^{46}$

The possibility of extrathymic T-cell development was explored in Xenopus by including PCR controls of gut tissue at the time of thymectomy in addition to the adult gut from normal and thymectomized frogs (Figure 1). Extrathymic T-cell development has been described in the mouse gut ${ }^{47}$ and may even be stimulated by thymic ablation. ${ }^{48}$ Highly organized mucosal lymphoid structures such as Peyer's patches do not exist in poikilothermic vertebrates such as Xenopus, ${ }^{49}$ yet a suggestion of gut $\mathrm{T}$ lymphopoiesis has been described from bony fish. ${ }^{50}$ Tadpoles showed no TCR expression in the gut at the age of thymectomy, nor was TCR expression seen in the adult gut of larval thymectomized frogs. While extrathymic routes of T-cell development are possible in the frog, our data suggest that these are at best minor relative to thymic for gut seeding, and that thymectomy does not force extrathymic developmental programs.

Some "natural" gut IgA in mouse has been thought to be from T-independent B-1 cells. ${ }^{51}$ The specificity of gut IgA was later shown to be less "natural" and actually driven by specific antigens of the gut microbial symbionts and food. ${ }^{9}$ Moreover, use of TCR $\beta / \delta$ double knockout transgenic mice ensured that $\mathrm{T}$ cells from thymus or elsewhere were not responsible for this phenomenon, nor was any "bystander" contribution of their lymphokines. ${ }^{52}$ They further showed that this response was at least in part due to $\mathrm{B} 1$ peritoneal cells in mice. ${ }^{9}$ These findings seem consistent with the lack of effect that thymectomy has on mucosal IgX in the present study. Yet in mammals, there is plenty of evidence for T-dependence in gut IgA too. Most human IgA-switched plasma cells in the lamina propria show evidence of somatic hypermutation, presumably from a germinal center event with T-cell help. ${ }^{53,54}$ Moreover, most ( $\sim 80 \%)$ plasma cells in the gut were found to be antigen specific and not polyreactive. ${ }^{55}$ AID transgenic mice defective of somatic hypermutation but still competent to make IgA exhibited greater colony counts of small intestinal flora, germinal center hyperplasia, and susceptibility to Yersinia enterocolitica. ${ }^{56}$ This suggests T-dependent, germinal center processes do shape the gut flora through the specific IgA generated against it in mice. Deep sequencing of IgA rearrangements in $\mathrm{CD}^{-1-}$ mice showed evidence for T-dependent somatic hypermutation in aged mice, thus the relatively young age of the frogs in this study could be a factor. ${ }^{8}$ We did not find evidence corroborating such $\mathrm{T}$ dependences in the amphibian, but recognize that this is one relatively small study in a captive population.

\section{Evolution of mucosal antibody isotypes and IgA}

Recent data now allow more rigorous studies of the natural history of tetrapod immunoglobulin genes. Sequence similarities and predicted structural resemblance to IgM originally suggested that IgX might be the functional analog, but not the ortholog of IgA. ${ }^{16,17}$ The IgA of Aves appears to be a mucosal functional analog of mammalian $\operatorname{Ig} \mathrm{A}^{57}$ and there is high sequence identity that suggests orthology. ${ }^{58}$ However, there are four $\mathrm{C}$ domains in avian IgA suggesting a deletion occurred to yield the mammalian IgA of three. ${ }^{59}$ The incomplete evolutionary loss of the $\mathrm{C} \alpha 2$ domain present in IgX and avian IgA could have given rise to the hinge region in mammalian IgA before the divergence of monotremes and the therian marsupial and placental mammals. ${ }^{60}$

Although IgX has been hypothesized to be an ortholog of $\operatorname{IgA},{ }^{15,61}$ the phylogenetic analysis described here is the first to lend experimental support to the notion. The availability of more diverse tetrapod immunoglobulin sequences allowed us to make this analysis. The $\mathrm{CH} 1$ - and $\mathrm{CH} 2$-encoding exons of the IgX gene may have been derived from the IgY-encoding locus and the $\mathrm{CH} 3$ and $\mathrm{CH} 4$ from the IgM-encoding gene. ${ }^{62,63}$ These relationships will need to be retested as more amphibian, reptile, bird, and non-placental mammal genomes are sequenced. However, this scenario is consistent with the known synteny of the C-region-encoding genes in the immunoglobulin heavy chain loci of known tetrapod genomes (Supplementary Figure 4 online), where $\operatorname{IgX} / \mathrm{A}$ is in between the genes encoding the IgM/D- and IgY-encoding loci that birthed it. The locus in mammals has undergone duplicative expansions giving rise to subfunctionalization of IgG and IgE from IgY and a proliferation of sub-isotypes. ${ }^{64}$ Thus, the resulting IgY/IgM chimera IgX gave rise to (or perhaps should be synonymous with) IgA in endothermic vertebrates. This appears to be the second time vertebrate evolution has produced a dedicated mucosal immunoglobulin isotype (Figure 7), the first being IgZ/T that is unique to some teleost fish. ${ }^{65-67} \mathrm{IgX} / \mathrm{A}$ is the first mucosal isotype whose expression is controlled via AID-mediated class switch recombination, as IgZ/T is produced via deletional RAG-mediated V(D)J recombination similar to the rearrangement at the $\alpha / \delta$ T-cell receptor locus. ${ }^{68,69}$

In the frog, we find evidence for an ancient, T-independent, humoral mucosal response. We defined the gut flora of the 
a

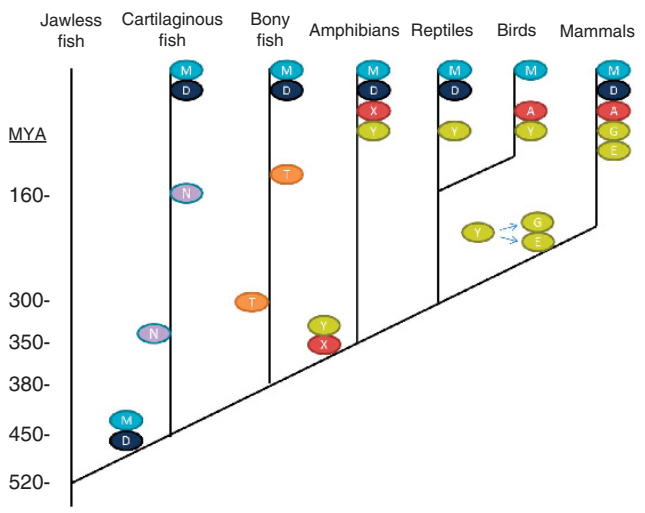

b

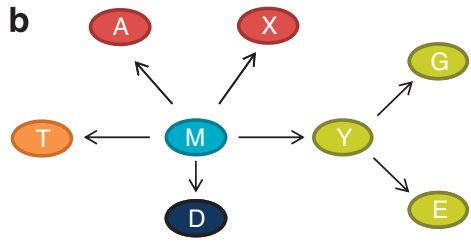

Previous consensus

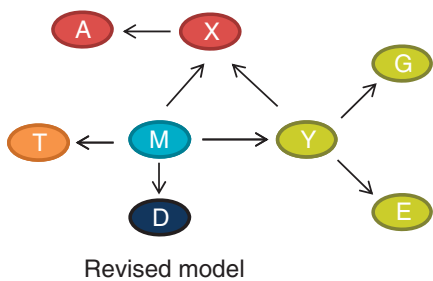

Figure 7 Model of immunoglobulin natural history with mucosal IgX/A emerging in early tetrapods. (a) Simplified phylogeny of vertebrates showing approximate emergence times of heavy chain isotypes. (b) This analysis suggests that the isotype previously described as IgX in amphibians may be orthologous to IgA of warm-blooded vertebrates (model adapted from M Flajnik's chapter of Fundamental Immunology, W. Paul editor). In addition to the isotypes extant in man, the immunoglobulin light chain-less IgNAR of cartilaginous fish and the mucosal IgZ/T of bony fish are also shown.

model amphibian X. laevis but found the bacterial communities of the stomach, small and large intestine to be unaffected by thymectomy. More representative phylogenetic analysis of the relationship between amphibian IgX and IgA of amniotic tetrapods shows their orthology, explaining the origin of human's most abundant immunoglobulin. Therefore, we conclude that the T-cell-independent IgA pathway is likely an ancient mechanism to manage the microbial symbionts of the gut and other mucosal surfaces. More comparative studies must resolve the (convergent?) functional relationship between the two vertebrate mucosal isotypes IgZ/T and IgX/A.

\section{METHODS}

Animals. X. laevis was used as a model for the tetrapod vertebrate immune system. Outbred frogs were initially purchased from Xenopus Express (Brooksville, FL). Subsequent generations were bred in-house using human chorionic gonadotropin hormone to prime for egg and sperm maturation (Sigma-Aldrich, St Louis, MO). Frogs were maintained at the Texas A\&M Comparative Medicine Program facility. They were housed in two separate but similar recirculating rack systems (Techniplast, Buguggiate, Italy) on a 12-h light cycle. Frogs were moved from an antigen-free system to a "DNP-KLH exposed" system upon first immunization. Adults were fed a sinking pellet and tadpoles a powder diet (Xenopus Express). All husbandry, surgery, and immunization protocols were approved by the Texas A\&M Institutional Animal Care and Use Committee (AUP 2008-33). Post-metamorphosis frogs were microchipped (Avid, Norco, CA) and assigned to gut microbiota harvest or immunization protocols.

Thymectomy. Frogs were thymectomized 9 days post fertilization through microscopic cauterization, adapting the protocol devised by Horton. ${ }^{19}$ The surgery was performed under a dissecting microscope using a micro-cautery apparatus originally designed for insect stylectomy (http://aphidzapper.com), delivering a VHF pulse of 10-ms duration and power of $10 \mathrm{~W}$ via an abraded tungsten wire to the target tissue. Tadpoles were anesthetized using a $300 \mathrm{mgl}^{-1} \mathrm{MS} 222$ (tricaine methanesulfonate, Argent, Redmond, WA) bath for 2-10 min, before placing on a wet cool cheesecloth over a grounded metal plate stage. The thymus was then burned with one pulse on each side. The thymus is located bilaterally caudal and medial to the eyes and lateral to the dark central nervous system (Figure 1a). The tadpole was transferred to an ice bath immediately after thymic ablation to cool the animal for $3 \mathrm{~s}$ before being allowed to recover in an aerated tank with $80 \mathrm{mgl}^{-1}$ carbenicillin (and no MS-222) to prevent superficial infections. After $24 \mathrm{~h}$, tadpoles were transferred back to the primary recirculating Xenoplus systems (with no antibiotic). Three days after surgery, tadpoles were monitored for any regrowth of the thymus by visual inspection for the melanized organ under the microscope ( $20 \%$ of frogs that recover from surgery). The monitoring continued every 4-6 days for 1 month post surgery. Tadpoles with thymic regrowth owing to incomplete thymectomy were euthanized.

PCR validation of thymectomy. Frogs thymectomized as tadpoles were checked for the presence of TCR $\alpha, \beta$, and $\delta$ messenger RNA using PCR at least 6 months post surgery (they undergo metamorphosis in the second month, Supplementary Table S1 online). The more exposed tarsal veins associated with digits two and three were bled for 100-500 $\mu \mathrm{l}$ with $1-\mathrm{ml}$ syringes and 28 gauge needles. Additional checks for TCR expression were performed on the gut of 9-day tadpoles, the gut of thymectomized adult, and the gut of normal adult. PCR was performed on complementary DNA prepared from RNA prepared from whole peripheral blood, owing to the small volumes of blood collected using RNAeasy preparations (Qiagen, Valencia, CA) with on-column genomic DNA digestion. First-strand complementary DNA was synthesized using random hexamer priming with Superscript III (Invitrogen, Carlsbad, CA) according to the manufacturer's protocol. PCR amplification was performed using oligonucleotide primers designed for constant domain genes of TCR $\alpha, \beta, \delta$, and $\beta_{2} \mathrm{M}$ (Supplementary Table $\mathbf{S} 2$ online). Gotaq polymerase (Promega, Madison, WI) and $50 \mathrm{ng}$ template were used during these PCR for 35 cycles for blood and $2 \mu \mathrm{g}$ template for 39 cycles for intestine. Primer sets for TCR $\alpha$ and $\beta_{2} \mathrm{~m}$ annealed at $52^{\circ} \mathrm{C}$ and $\beta$ and $\delta$ at $58^{\circ} \mathrm{C}$.

DNA preparation and 16S rRNA gene sequencing. Gut contents were sampled from normal and thymectomized frogs at three anatomical sites: stomach, small intestine, and large intestine (as shown in Supplementary Figure $\mathbf{S 1}$ online). Bolus and chyme were scraped from longitudinally opened organs and collected as $\sim 300-\mu \mathrm{l}$ samples for DNA isolation via phenol-chloroform-isoamyl alcohol extraction. ${ }^{34}$ Bacterial tag-encoded FLX-titanium amplicon pyrosequencing (bTEFAP) was performed similarly as described previously at the Research and Testing Laboratory, Lubbock, $\mathrm{TX},{ }^{70}$ but based on the $\mathrm{V} 4-\mathrm{V} 6$ region (E. coli position 
$530-1,100)$ of the $16 \mathrm{~S}$ rRNA gene, with primers forward $530 \mathrm{~F}$ and reverse 1100R (Supplementary Table S2 online). Briefly, the DNA concentration was determined using a Nanodrop spectrophotometer (Nyxor Biotech, Paris, France). A 100-ng ( $1 \mu \mathrm{l})$ aliquot of each DNA sample was used for a 50- $\mu$ l PCR reaction. HotStarTaq Plus Master Mix Kit (Qiagen) was used for PCR under the following conditions: $94^{\circ} \mathrm{C}$ for $3 \mathrm{~min}$ followed by 32 cycles of $94^{\circ} \mathrm{C}$ for $30 \mathrm{~s} ; 60^{\circ} \mathrm{C}$ for $40 \mathrm{~s}$; and $72^{\circ} \mathrm{C}$ for $1 \mathrm{~min}$; and a final elongation step at $72^{\circ} \mathrm{C}$ for $5 \mathrm{~min}$. A secondary PCR was performed for FLX (Roche, Nutley, NJ) amplicon sequencing under the same conditions by using designed special fusion primers with different tag sequences as LinkerA-Tags-530F and LinkerB-1100R. The use of a secondary PCR prevents amplification of any potential bias that might be caused by inclusion of tag and linkers during initial template amplification reactions. After secondary PCR, all amplicon products from the different samples were mixed in equal volumes, and purified using Agencourt Ampure beads (Agencourt, Danvers, MA).

Gut flora analysis. Raw sequence data were screened, trimmed, filtered, denoised, and chimera depleted with default settings using the QIIME pipeline version 1.4.0 (http://qiime.sourceforge.net) ${ }^{71}$ and with USEARCH using the operational taxonomic unit pipeline (www.drive5. com). Operational taxonomic units were defined as sequences with at least 97\% similarity using QIIME. For classification of sequences on a genus level, the naive Bayesian classifier within the Ribosomal Database Project (RDP, v10.28, East Lansing, MI) was used. The compiled data were used to determine the relative percentages of bacteria for each individual sample, 6 frogs by 3 samples each for a total of 18 . To account for unequal sequencing depth across samples, subsequent analysis was performed on a randomly selected subset of 1,800 sequences per sample. This number was chosen to avoid exclusion of samples with lower number of sequence reads from further analysis. Alpha diversity (i.e., rarefaction) and beta diversity measures were calculated and plotted using QIIME. Differences in microbial communities between disease groups were investigated using the phylogeny-based unweighted Unifrac distance metric. This analysis measures the phylogenetic distance among bacterial communities in a phylogenetic tree, and thereby provides a measure of similarity among microbial communities present in different biological samples.

IgX phylogenetics. Amino-acid sequences of tetrapod immunoglobulin heavy chain constant regions were compiled and aligned in BioEdit with ClustalW employing gap opening penalties of 10 and gap extension penalties of 0.1 for pairwise alignments and then 0.2 for multiple alignments with the protein-weighting matrix of Gonnett or Blossum. ${ }^{72,73}$ These alignments were then heavily modified by hand. MEGA was used to infer the phylogenetic relationships of the immunoglobulin heavy chain constant genes. Evolutionary distances were computed using the Dayhoff matrix ${ }^{74}$ and 509 column positions in the 56 selected sequences. Several tree-building algorithms were employed, including a consensus neighbor-joining tree made from 1,000 bootstrap replicates.

Immunizations. For immunization, two routes of administration were used. Four frogs received IC (frogs have no peritoneal cavity) and four oral (p.o.) immunization. We used an oral gavage needle to deliver conjugated DNP-KLH (Calbiochem, San Diego, CA) for mucosal immunization as previously described for X. laevis. ${ }^{15}$ There were two normal and two thymectomized frogs in each group for a total of eight frogs. Frogs orally immunized received $2.5 \mathrm{mg} \mathrm{DNP} / \mathrm{KLH}$ with $10 \mu \mathrm{g}$ cholera toxin as adjuvant three times, each at weekly intervals. Animals in the IC group received $200 \mu \mathrm{g}$ of antigen with equal volume $(200 \mu \mathrm{l})$ of Freund's complete adjuvant once and Freund's incomplete adjuvant twice, at weekly intervals.

Lymphocyte isolation and culture. Three weeks after the last boost, we euthanized frogs with an MS-222 overdose and decapitation. Spleens were removed and cells dissociated by scraping the organs over a wire mesh inundated with amphibian-adjusted phosphate-buffered saline (PBS). The intestine was excised below the stomach and above the rectum. We removed mucous, chime, and fecal matter by physically scraping and flushing the organ with PBS. The intestine was cut into smaller pieces $\left(\sim 0.5 \mathrm{~cm}^{2}\right)$ and placed in PBS. We then added $2 \%$ collagenase (Calbiochem) in amphibian PBS to aid in isolation of the intestinal lymphocytes. We allowed the intestine to digest for $120 \mathrm{~min}$ at room temperature. It was vortexed initially and once every $30 \mathrm{~min}$. The intestine was strained through a $100 \mu$ m nylon cell strainer (BD Falcon, San Jose, $\mathrm{CA}$ ) at the end of the $120 \mathrm{~min}$. Lymphocytes were isolated from the washed supernatant of the intestine and from the cells isolated from the spleen with Lymphocyte Separation Medium (Mediatech, Manassas, VA). All intestine and spleen cells isolated were counted (Supplementary Figure S2 online) and cultured in 24-well plates containing $250 \mu \mathrm{l}$ of L-15 media with $10 \%$ fetal calf serum at a density of $2.3 \times 10^{6}$ cells per $\mathrm{ml}$ for the spleen cells and $1.0 \times 10^{6}$ cells per $\mathrm{ml}$ for the intestinal cells. Cultures were incubated at humidified $28^{\circ} \mathrm{C}$ and $5 \% \mathrm{CO}_{2}$. On the third day, supernatant was collected and new media was added to maintain a volume of $250 \mu \mathrm{l}$ per well. On the sixth day, supernatant was once again collected and pooled with the day 3 collections.

Enzyme-linked immunosorbent assay. We used ELISAs to determine the total (non-antigen specific) and antigen-specific levels of amphibian mucosal antibody isotype IgX produced in response to DNP-KLH. Serial dilutions from $1 / 10$ to $1 / 10^{11}$ were made from the supernatants of the spleen and intestinal cultures. For the total immunoglobulin ELISAs, we added $100 \mu \mathrm{l}$ of each dilution to the well of a plate and incubated for $1 \mathrm{~h}$ at $37^{\circ} \mathrm{C}$. The plate was then washed 2 times with $200 \mu \mathrm{l}$ PBS, and $200 \mu \mathrm{l}$ of $2 \%$ casein in PBS was added as blocking solution. The plate was allowed to sit overnight at $4^{\circ} \mathrm{C}$, and was then washed 3 times with $200 \mu \mathrm{l}$ PBS. We added mouse anti-Xenopus IgX monoclonal 4110B3 (kind gifts of Martin Flajnik, University of Maryland at Baltimore and Louis Du Pasquier, University of Basel) to each well to obtain a volume of $100 \mu l{ }^{75}$ The plate was allowed to incubate at room temperature for $1 \mathrm{~h}$ and then was washed 4 times with $200 \mu \mathrm{l}$ PBS with $0.05 \%$ Tween-20 (PBS-T, Sigma, St Louis, MO). The wells received $100 \mu \mathrm{l}$ of anti-mouse IgG peroxidaseconjugated secondary antibody (Sigma) and were incubated for $1 \mathrm{~h}$ at room temperature. We then washed the plate 4 times with $200 \mu \mathrm{l}$ PBS-T and 1 time with PBS. A 3,3',5,5' -tetramethylbenzidine substrate solution was then added to each well and the reaction was allowed to take place for 3 min before being stopped with $2 \mathrm{M} \mathrm{H}_{2} \mathrm{SO}_{4}$. Plates were read at an optical density of $450 \mathrm{~nm}$ in a BioRad iMark Microplate Reader (Hercules, CA). We used the $10^{4}$ dilution of supernatant for all trials. The antigen-specific ELISAs used a similar protocol, except $100 \mu \mathrm{l}$ of $10 \mu \mathrm{g} \mathrm{ml}^{-1} \mathrm{DNP}-\mathrm{KLH}$ was added to each well for initial coating. After blocking overnight, serial dilutions of the sample supernatant were added and allowed to incubate for $2 \mathrm{~h}$ at $37^{\circ} \mathrm{C}$. The remaining protocol was the same as that for total $\operatorname{IgX}$. We assayed wells in triplicate, showed the s.e.m. and employed a Student's $t$-test.

SUPPLEMENTARY MATERIAL is linked to the online version of the paper at http://www.nature.com/mi

\section{ACKNOWLEDGMENTS}

This work was supported by the NIH through a K22 award to MFC (AI56963) and a graduate student grant from the Texas A\&M College of Veterinary Medicine to SM. AG was supported by the National Center for Research Resources and the Office of Research Infrastructure Programs (ORIP) of the National Institutes of Health through Grant Number 5 T35 RR019530-07. We appreciate the expert training in tadpole thymectomy provided by Martin Flajnik.

\section{DISCLOSURE}

The authors declare no conflict of interest.

(C) 2013 Society for Mucosal Immunology 


\section{REFERENCES}

1. Cooper, M.D., Raymond, D.A., Peterson, R.D., South, M.A. \& Good, R.A. The functions of the thymus system and the bursa system in the chicken. J. Exp. Med. 123, 75-102 (1966).

2. Criscitiello, M.F., Ohta, Y., Saltis, M., McKinney, E.C. \& Flajnik, M.F. Evolutionarily conserved TCR binding sites, identification of T cells in primary lymphoid tissues, and surprising trans-rearrangements in nurse shark. J. Immunol. 184, 6950-6960 (2010).

3. Weidberg, H., Shpilka, T., Shvets, E., Abada, A., Shimron, F. \& Elazar, Z LC3 and GATE-16 N termini mediate membrane fusion processes required for autophagosome biogenesis. Dev. Cell 20, 444-454 (2011a)

4. Weidberg, H., Shvets, E. \& Elazar, Z. Biogenesis and cargo selectivity of autophagosomes. Annu. Rev. Biochem. 80, 125-156 (2011b).

5. Saftig, P. \& Klumperman, J. Lysosome biogenesis and lysosomal membrane proteins: trafficking meets function. Nat. Rev. Mol. Cell Biol. 10, 623-635 (2009).

6. Crabbe, P.A., Nash, D.R., Bazin, H., Eyssen, H. \& Heremans, J.F. Immunohistochemical observations on lymphoid tissues from conventional and germ-free mice. Lab. Invest. 22, 448-457 (1970).

7. Ebersole, J.L., Taubman, M.A. \& Smith, D.J. Thymic control of secretory antibody responses in the rat. J. Immunol. 123, 19-24 (1979).

8. Lindner, C. et al. Age, microbiota, and T cells shape diverse individual IgA repertoires in the intestine. J. Exp. Med. 209, 365-377 (2012).

9. Macpherson, A.J., Gatto, D., Sainsbury, E., Harriman, G.R., Hengartner, H. \& Zinkernagel, R.M. A primitive T cell-independent mechanism of intestinal mucosal lgA responses to commensal bacteria. Science $\mathbf{2 8 8}$, 2222-2226 (2000).

10. Macpherson, A.J. \& Slack, E. The functional interactions of commensal bacteria with intestinal secretory IgA. Curr. Opin. Gastroenterol. 23, 673-678 (2007).

11. Fritz, J.H. et al. Acquisition of a multifunctional lgA+ plasma cell phenotype in the gut. Nature 481, 199-203 (2012).

12. Li, J. et al. B lymphocytes from early vertebrates have potent phagocytic and microbicidal abilities. Nat. Immunol. 7, 1116-1124 (2006).

13. Korolchuk, V.I. et al. Lysosomal positioning coordinates cellular nutrient responses. Nat. Cell Biol. 13, 453-460 (2011).

14. Horton, J.D. et al. T-cell and natural killer cell development in thymectomized Xenopus. Immunol. Rev. 166, 245-258 (1998).

15. Du, C.C., Mashoof, S.M. \& Criscitiello, M.F. Oral immunization of the African clawed frog (Xenopus laevis) upregulates the mucosal immunoglobulin IgX. Vet. Immunol. Immunopathol. 145, 493-498 (2012).

16. Mussmann, R., Du, P.L. \& Hsu, E. Is Xenopus IgX an analog of IgA? Eur. J. Immunol. 26, 2823-2830 (1996a).

17. Mussmann, R., Wilson, M., Marcuz, A., Courtet, M. \& Du, P.L. Membrane exon sequences of the three Xenopus Ig classes explain the evolutionary origin of mammalian isotypes. Eur. J. Immunol. 26, 409-414 (1996b).

18. Tochinai, S. Demonstration of thymus-independent immune system in Xenopus laevis. Response to polyvinylpyrrolidone. Immunology 31 , 125-128 (1976).

19. Horton, J.D. \& Manning, M.J. Response to skin allografts in Xenopus laevis following thymectomy at early stages of lymphoid organ maturation. Transplantation 14, 141-154 (1972).

20. Kuczynski, J. et al. Direct sequencing of the human microbiome readily reveals community differences. Genome Biol. 11, 210 (2010).

21. Nocker, A., Burr, M. \& Camper, A.K. Genotypic microbial community profiling: a critical technical review. Microb. Ecol. 54, 276-289 (2007).

22. Huse, S.M., Dethlefsen, L., Huber, J.A., Mark Welch, D., Relman, D.A. \& Sogin, M.L. Exploring microbial diversity and taxonomy using SSU rRNA hypervariable tag sequencing. PLoS Genet. 4, e1000255 (2008).

23. Nordentoft, S., Molbak, L., Bjerrum, L., De Vylder, J., Van Immerseel, F. \& Pedersen, $K$. The influence of the cage system and colonisation of Salmonella enteritidis on the microbial gut flora of laying hens studied by T-RFLP and 454 pyrosequencing. BMC Microbiol. 11, 187 (2011).

24. van Kessel, M.A. et al. Pyrosequencing of 16 S rRNA gene amplicons to study the microbiota in the gastrointestinal tract of carp (Cyprinus carpio L.). AMB Express 1, 41 (2011).

25. Robert, J. \& Ohta, Y. Comparative and developmental study of the immune system in Xenopus. Dev. Dyn. 238, 1249-1270 (2009).

26. Lauer, A., Simon, M.A., Banning, J.L., Lam, B.A. \& Harris, R.N. Diversity of cutaneous bacteria with antifungal activity isolated from female four-toed salamanders. ISME J. 2, 145-157 (2008).
27. Gossling, J., Loesche, W.J. \& Nace, G.W. Large intestine bacterial flora of nonhibernating and hibernating leopard frogs (Rana pipiens). Appl. Environ. Microbiol. 44, 59-66 (1982).

28. Banas, J.A., Loesche, W.J. \& Nace, G.W. Classification and distribution of large intestinal bacteria in nonhibernating and hibernating leopard frogs (Rana pipiens). Appl. Environ. Microbiol. 54, 2305-2310 (1988).

29. Detkova, E.N., Zaichikova, M.V. \& Kevbrin, V.V. [Physiology and biochemistry of alkaliphilic anaerobic hydrolytic bacterium Alkaliflexus imshenetskii]. Mikrobiologiia 78, 310-316 (2009).

30. Mulder, I.E. et al. Environmentally-acquired bacteria influence microbial diversity and natural innate immune responses at gut surfaces. BMC Biol. 7, 79 (2009).

31. Zhou, J. et al. Molecular and biochemical characterization of a novel xylanase from the symbiotic Sphingobacterium sp. TN19. Appl. Microbiol. Biotechnol. 85, 323-333 (2009).

32. Rawls, J.F., Mahowald, M.A., Ley, R.E. \& Gordon, J.I. Reciprocal gut microbiota transplants from zebrafish and mice to germ-free recipients reveal host habitat selection. Cell 127, 423-433 (2006).

33. Eckburg, P.B. et al. Diversity of the human intestinal microbial flora. Science 308, 1635-1638 (2005).

34. Suchodolski, J.S., Ruaux, C.G., Steiner, J.M., Fetz, K. \& Williams, D.A. Application of molecular fingerprinting for qualitative assessment of smallintestinal bacterial diversity in dogs. J. Clin. Microbiol. 42, 4702-4708 (2004).

35. Guy-Grand, D., Griscelli, C. \& Vassalli, P. Peyer's patches, gut IgA plasma cells and thymic function: study in nude mice bearing thymic grafts. J. Immunol. 115, 361-364 (1975).

36. Brown, J.F. \& Balish, E. Gastrointestinal microecology of BALB/c nude mice. Appl. Environ. Microbiol. 36, 144-159 (1978).

37. Lee, Y.K. \& Mazmanian, S.K. Has the microbiota played a critical role in the evolution of the adaptive immune system? Science $330,1768-1773$ (2010).

38. McFall-Ngai, M. Adaptive immunity: care for the community. Nature 445, 153 (2007).

39. Gravenor, I., Horton, T.L., Ritchie, P., Flint, E. \& Horton, J.D. Ontogeny and thymus-dependence of $T$ cell surface antigens in Xenopus: flow cytometric studies on monoclonal antibody-stained thymus and spleen. Dev. Comp. Immunol. 19, 507-523 (1995).

40. Horton, T.L., Ritchie, P., Watson, M.D. \& Horton, J.D. NK-like activity against allogeneic tumour cells demonstrated in the spleen of control and thymectomized Xenopus. Immunol. Cell Biol. 74, 365-373 (1996).

41. Horton, T.L., Ritchie, P., Watson, M.D. \& Horton, J.D. NK cell evolution: studies on Xenopus. Biochem. Soc. Trans. 25, $263 S$ (1997).

42. Nagata, S. \& Cohen, N. Specific in vivo and nonspecific in vitro alloreactivities of adult frogs (Xenopus laevis) that were thymectomized during early larval life. Eur. J. Immunol. 13, 541-545 (1983).

43. Chida, A.S., Goyos, A. \& Robert, J. Phylogenetic and developmental study of CD4, CD8 alpha and beta T cell co-receptor homologs in two amphibian species, Xenopus tropicalis and Xenopus laevis. Dev. Comp. Immunol. 35, 366-377 (2011).

44. Soloff, R.S., Wang, T.G., Lybarger, L., Dempsey, D. \& Chervenak, R. Transcription of the TCR-beta locus initiates in adult murine bone marrow. J. Immunol. 154, 3888-3901 (1995).

45. Spiess, S., Kuhrober, A., Schirmbeck, R., Arden, B. \& Reimann, J. Diversity of functional T-cell receptor delta-chain transcripts from bone marrow cells of athymic nude mice. Immunology 78, 252-259 (1993).

46. Horton, J., Horton, T., Ritchie, P., Gravenor, I., Gartland, L. \& Cooper, M. Use of Monoclonal Antibodies to Demonstrate Absence of T Cell Development in Early-Thymectomized Xenopus (Developmental and Comparative Immunology, Wageningen, The Netherlands, 1994).

47. Guy-Grand, D., Cerf-Bensussan, N., Malissen, B., Malassis-Seris, M., Briottet, C. \& Vassalli, P. Two gut intraepithelial CD8+ Iymphocyte populations with different $T$ cell receptors: a role for the gut epithelium in T cell differentiation. J. Exp. Med. 173, 471-481 (1991).

48. Guy-Grand, D. et al. Extrathymic T cell lymphopoiesis: ontogeny and contribution to gut intraepithelial lymphocytes in athymic and euthymic mice. J. Exp. Med. 197, 333-341 (2003).

49. Shields, J.W. The functional evolution of GALT: a review. Lymphology 33, 47-57 (2000).

50. Rombout, J.H., Abelli, L., Picchietti, S., Scapigliati, G. \& Kiron, V. Teleost intestinal immunology. Fish Shellfish Immunol. 31, 616-626 (2011). 
51. Kroese, F.G., Ammerlaan, W.A., Deenen, G.J., Adams, S., Herzenberg, L.A. \& Kantor, A.B. A dual origin for IgA plasma cells in the murine small intestine. Adv. Exp. Med. Biol. 371A, 435-440 (1995).

52. Wetzel, G.D. Interleukin 5 regulation of peritoneal Ly-1 B lymphocyte proliferation, differentiation and autoantibody secretion. Eur. J. Immunol. 19, 1701-1707 (1989)

53. Dunn-Walters, D.K., Boursier, L. \& Spencer, J. Hypermutation, diversity and dissemination of human intestinal lamina propria plasma cells. Eur. J. Immunol. 27, 2959-2964 (1997a).

54. Dunn-Walters, D.K., Isaacson, P.G. \& Spencer, J. Sequence analysis of human IgVH genes indicates that ileal lamina propria plasma cells are derived from Peyer's patches. Eur. J. Immunol. 27, 463-467 (1997b).

55. Benckert, J. et al. The majority of intestinal lgA+ and lgG+ plasmablasts in the human gut are antigen-specific. J. Clin. Invest. 121, 1946-1955 (2011)

56. Wei, M., Shinkura, R., Doi, Y., Maruya, M., Fagarasan, S. \& Honjo, T. Mice carrying a knock-in mutation of Aicda resulting in a defect in somatic hypermutation have impaired gut homeostasis and compromised mucosal defense. Nat. Immunol. 12, 264-270 (2011).

57. Mansikka, A. Chicken IgA H chains. Implications concerning the evolution of H chain genes. J. Immunol. 149, 855-861 (1992).

58. Choi, S.Y., Suh, K.S., Choi, D.E. \& Lim, B.J. Morphometric analysis of podocyte foot process effacement in IgA nephropathy and its association with proteinuria. Ultrastruct. Pathol. 34, 195-198 (2010).

59. Aveskogh, M. \& Hellman, L. Evidence for an early appearance of modern post-switch isotypes in mammalian evolution; cloning of lgE, lgG and IgA from the marsupial Monodelphis domestica. Eur. J. Immunol. 28, 2738-2750 (1998).

60. Vernersson, M., Belov, K., Aveskogh, M. \& Hellman, L. Cloning and structural analysis of two highly divergent IgA isotypes, IgA1 and IgA2 from the duck billed platypus, Ornithorhynchus anatinus. Mol. Immunol. 47, 785-791 (2010)

61. Sun, Y., Wei, Z., Li, N. \& Zhao, Y. A comparative overview of immunoglobulin genes and the generation of their diversity in tetrapods. Dev. Comp. Immunol. advance online publication, 23 February 2012 (in press).

62. Deza, F.G., Espinel, C.S. \& Beneitez, J.V. A novel IgA-like immunoglobulin in the reptile Eublepharis macularius. Dev. Comp. Immunol. 31, 596-605 (2007).
63. Wei, Z. et al. Expression of lgM, lgD, and lgY in a reptile, Anolis carolinensis. J. Immunol. 183, 3858-3864 (2009).

64. Warr, G.W., Magor, K.E. \& Higgins, D.A. IgY: clues to the origins of modern antibodies. Immunol. Today 16, 392-398 (1995).

65. Danilova, N., Bussmann, J., Jekosch, K. \& Steiner, L.A. The immunoglobulin heavy-chain locus in zebrafish: identification and expression of a previously unknown isotype, immunoglobulin Z. Nat. Immunol. 6, 295-302 (2005).

66. Hansen, J.D., Landis, E.D. \& Phillips, R.B. Discovery of a unique Ig heavychain isotype ( $\lg T)$ in rainbow trout: implications for a distinctive B cell developmental pathway in teleost fish. Proc. Natl Acad. Sci. USA 102, 6919-6924 (2005)

67. Zhang, Y.A. et al. IgT, a primitive immunoglobulin class specialized in mucosal immunity. Nat. Immunol. 11, 827-835 (2010).

68. Flajnik, M.F. The last flag unfurled? A new immunoglobulin isotype in fish expressed in early development. Nat. Immunol. 6, 229-230 (2005).

69. Salinas, I., Zhang, Y.A. \& Sunyer, J.O. Mucosal immunoglobulins and B cells of teleost fish. Dev. Comp. Immunol. 35, 1346-1365 (2011).

70. Handl, S., Dowd, S.E., Garcia-Mazcorro, J.F., Steiner, J.M. \& Suchodolski, J.S. Massive parallel 16S rRNA gene pyrosequencing reveals highly diverse fecal bacterial and fungal communities in healthy dogs and cats. FEMS Microbiol. Ecol. 76, 301-310 (2011).

71. Caporaso, J.G. et al. QIIME allows analysis of high-throughput community sequencing data. Nat. Methods 7, 335-336 (2010).

72. Hall, T.A. BioEdit: a user-friendly biological sequence alignment editor and analysis program for Windows 95/98/NT. Nucleic Acids Symp. Ser. 41, 95-98 (1999).

73. Tamura, K., Dudley, J., Nei, M. \& Kumar, S. MEGA4: Molecular Evolutionary Genetics Analysis (MEGA) software version 4.0. Mol. Biol. Evol. 24, 1596-1599 (2007).

74. Schwarz, R. \& Dayhoff, M. Matrices for detecting distant relationships. In Atlas of Protein Sequences (Dayhoff M., ed). Columbia, MD, pp 353-358 (National Biomedical Research Foundation, 1979).

75. Hsu, E. \& Du Pasquier, L. Studies on Xenopus immunoglobulins using monoclonal antibodies. Mol. Immunol. 21, 257-270 (1984).

76. Flajnik, M. Evolution of the immune system. In Fundamental Immunology (Paul W.E. (ed)). Lippincott Williams \& Wilkins: Wolters Kluwer, Philadelphia, 2008, pp 56-124. 